\title{
Requirement of Intravenous Fluid and Insulin in the Management of Diabetic Ketoacidosis to Overcome the Crisis
}

\author{
MUHAMMAD IQBAL QASIM ${ }^{1}$, MUHAMMAD MUJTABA HASHIR ${ }^{2}$, SHAHEERA BATOOL ${ }^{3}$, NISAR KHAN SAJID ${ }^{4}$, WAHEED \\ IQBAL ${ }^{5}$, MUHAMMAD SHAHBAZ ASHRAF $^{6}$ \\ ${ }^{1}$ Assistant Professor of Medicine Frontier Medical College Abbottabad \\ ${ }^{2}$ Associate Professor of Medicine, Nishtar Medical University Hospital Multan \\ ${ }^{3}$ Assistant Professor of Biochemistry, CMH Multan Institute of Medical Science Multan \\ ${ }^{4}$ Associate Professor of Pediatric Medicine, Aziz Fatima Medical and Dental College, Faisalabad \\ ${ }^{5}$ Associate Professor Department of Medicine Divisional Headquarters Teaching Hospital Mirpur /Mohtarma Benazir Bhutto Shaheed \\ Medical College Mirpur \\ ${ }^{6}$ Senior Registrar, Department of Medicine Mayo Hospital, Lahore \\ Corresponding author: Muhammad lqbal Qasim, Email: driqbalqasim2000@gmail.com, Contact: +923339223068
}

\begin{abstract}
Objective: The aim of this study is to determine the requirement of intravenous fluid and insulin in the treatment of diabetic ketoacidosis to overcome the crisis.

Study Design: Cross-sectional

Place and Duration:Study was conducted at Medicine department of King Abdullah Teaching Hospital Mansehra and Nishtar Medical University Hospital Multan for six months duration from $15^{\text {th }}$ December, 2020 to $15^{\text {th }}$ June, 2020.

Methods: Total seventy patients of both genders were presented in this study. Patients were aged between 1865 years. Patients details demographics age, sex and body mass index were recorded after taking written consent. Patients had diabetic ketoacidosis were presented in this study. Frequency of intravenous fluid and insulin were assessed to overcome the crisis of diabetic ketoacidosis by using portland protocol. Complete data was analyzed by SPSS 22.0 version.

Results: Mean age of the patients was $30.51 \pm 7.41$ years with mean BMI $25.18 \pm 3.48 \mathrm{~kg} / \mathrm{m}^{2} .38(54.3 \%)$ cases were females and $32(45.7 \%)$ patients were males. $42(60 \%)$ cases had previous history of diabetes and the rest28 (40\%) were newly diagnosed.Prevalence of DKA was more common in low socio-economic status found in $39(55.7 \%)$ cases. Vomiting was the most common symptom found in $35(50 \%)$ cases, followed by infection of insulin doses $18(25.7 \%)$ cases, abdominal pain found in $15(21.43 \%)$ patients. $32(45.7 \%)$ patients who had increased diuresis received $11 \mathrm{~L}$ of fluid in the treatment of DKA, 91-100 units of insulin were given to $18(25.71 \%)$ cases, and for management of acidosis 24 (34.3\%) patients took 2-days. There was no any mortality found in this study.

Conclusion:We concluded in this study that early diagnosed of diabetic ketoacidosis is necessary for treatment. Removal of adequate fluid was necessary to avoid mortality.

Keywords:Intravenous fluid, Insulin, Diabetic ketoacidosis, Diabetes
\end{abstract}

\section{INTRODUCTION}

In hyperglycemic state of diabetes mellitus, there is acute metabolic decompensation and very prevalent in this state is diabetic ketoacidosis[1-2] . It frequently happens among people of diabetes mellitus who have stopped captivating insulin or have any form of infection. DKA also developed in previously undiagnosed patients. Therefore, we evaluated seventy DKA patients to determine their treatment needs and eventually required of fluids and I / V insulin [3-4]. Treatment comprised of addressing acidosis, electrolyte imbalance, dehydration and treating the causal agent i.e., antibiotic for infection.[5]

The major triggers or causes of DKA in recently found diabetics are missed doses of insulin, and infections in people identified with diabetes. The basic mechanism driving this is insulin insufficiency. Insulin doses were disregarded because insulin was not available or available to low-income individuals in rural regions[6-7]. The knowledge among low-income individuals found to be below level and responsible for hospital admissions in all patients who are diabetes and established DKA. During the period of illness, when patients with type I and II progress vomiting and anorexia, patients generally need to check their urine ketone levels and blood sugar. They must boost their fluid intake. Insulin therapy should not be interrupted during this interval and doses should be adjusted[8-9]. Crystalloid is the optimal I.V fluid to treat dehydration compared to colloid, and retains hydration. Maximum of the participants require eleven liters of fluid to correct DKA and 48 hours are needed to correct DKA. In seven days; The maximum patients recovered [10-11]. Every participant needs an average of 4,12 liters of fluid IV, 72 insulin units and 60 moles of potassium during 1st 24 hours of hospitalization. In severe acidosis, bicarbonate is suggested. In poor nations, treatment forms part of general services were 6 percent. Mortality in the intensive care unit ranges from 2 percent to 5 percent in the late reporting of severe acidosis. In several centers, it has now fallen to $<1 \%$.

\section{MATERIAL AND METHODS}

This cross-sectional study was conducted at Medicine department of King Abdullah Teaching Hospital Mansehra and Nishtar Medical University Hospital Multan for six months duration from $15^{\text {th }}$ December, 2020 to $15^{\text {th }}$ June, 2020 and comprised of 70 diabetic patients. Patients 
details demographics were recorded after taking informed written consent. Patients had cardiac disease, less than 18 years of age and those did not give any written consent were excluded from this study.

Patients had diabetic ketoacidosis were presented in this study. Frequency of intravenous fluid and insulin were assessed to overcome the crisis of diabetic ketoacidosis by using portland protocol in the syringe pump in accordance with the Portland protocol,blood sugar was tested every hour, and insulin rate was adjusted. The intake-output chart shows whether 50 percent of the infused fluid has been maintained and also avoids overloading volumes. When bicarbonate exceeded $18 \mathrm{meq} / \mathrm{l}$ and $\mathrm{pH}>7.3$, DKA was considered resolving. Then, when patients could take food orally $(2 / 3$ of intermediate $\mathrm{NPH}$ insulin and $1 / 3$ of conventional insulin) split mixing schedule for subcutaneous insulin began. Two thirds of the entire daily dose in the morning and one third in the evening were provided. Complete data was analyzed by SPSS 22.0 version.

\section{RESULTS}

Mean age of the patients was $30.51 \pm 7.41$ years with mean BMI $25.18 \pm 3.48 \mathrm{~kg} / \mathrm{m}^{2}$. 38(54.3\%) cases were females and $32(45.7 \%)$ patients were males. $42(60 \%)$ cases had previous history of diabetes and the rest 28 (40\%) were newly diagnosed. Prevalence of DKA was more common in low socio-economic status found in 39 (55.7\%) cases. (table 1)

Table 1: Baseline details demographics of enrolled cases

\begin{tabular}{|l|l|l|}
\hline Variables & Frequency & \%age \\
\hline Mean age & $30.51 \pm 7.41$ & \\
\hline Mean BMI & $25.18 \pm 3.48$ & \\
\hline Gender & 32 & 45.7 \\
\hline Male & 38 & 54.3 \\
\hline Female & 28 & 40 \\
\hline History of diabetes & 42 & 60 \\
\hline Newly & 55.7 \\
\hline Old & 39 & 44.3 \\
\hline Socio-economic status & 31 & \\
\hline Low &
\end{tabular}

Vomiting was the most common symptom found in 35 $(50 \%)$ cases, followed by infection of insulin doses 18 $(25.7 \%)$ cases, abdominal pain found in 15 (21.43\%) patients, polyuria $12(17.14 \%)$ and polydipsia found in 11 (15.7\%).(table 2)

Table 1: Association of

\begin{tabular}{|l|l|l|}
\hline Variables & Frequency & \%age \\
\hline Symptoms & 35 & 50 \\
\hline Vomiting & 18 & 25.7 \\
\hline Infection of insulin doses & 15 & 21.43 \\
\hline Abdominal pain & 23 & 17.14 \\
\hline Polyuria & 11 & 15.7 \\
\hline Polydipsia &
\end{tabular}

Majority $32(45.7 \%)$ patients who had increased diuresis received $11 \mathrm{~L}$ of fluid in the treatment of DKA, 5 liters fluid were given to $14(20 \%)$ cases and maximum fluid was 16 liters which was given to rare cases $2(2.9 \%)$. (table 3)

Table 3: Amount of fluid for improvement of acidosis

Table 3: Amount of fluid for improvement of acidosis
\begin{tabular}{|l|l|l|}
\hline Variables & Frequency $(\mathrm{n}=70)$ & $\%$ age \\
\hline Amount of (NS) fluid (L) & 14 & 20 \\
\hline 5 & 5 & 7.14 \\
\hline 6 & 3 & 4.3 \\
\hline 7 & 6 & 10 \\
\hline 8 & 3 & 4.3 \\
\hline 9 & 5 & 7.14 \\
\hline 10 & 32 & 45.7 \\
\hline 11 & 2 & 2.9 \\
\hline 16 & & \\
\hline
\end{tabular}

91-100 units of insulin were given to majority 18 $(25.71 \%)$ cases and $141-150$ units were given to 3 (4.3\%).(table 4)

Table 4: Units of insulin for treatment of acidosis

\begin{tabular}{|l|l|l|}
\hline Variables & Frequency $(\mathrm{n}=70)$ & \%age \\
\hline Amount of (unit) & 13 & 18.6 \\
\hline $71-80$ & 10 & 14.3 \\
\hline $81-90$ & 18 & 25.71 \\
\hline $91-100$ & 5 & 7.14 \\
\hline $101-110$ & 6 & 8.6 \\
\hline $111-120$ & 5 & 7.14 \\
\hline $121-130$ & 6 & 8.6 \\
\hline $131-140$ & 3 & 4.3 \\
\hline $141-150$ & 4 & $5.7 \%$ \\
\hline $151-160$ & \multicolumn{2}{|l|}{} \\
\hline
\end{tabular}

Majority of the patients 24 (34.3\%) took 2-days for recovery of acidosis, followed by 1 -day in which 14 (20\%) cases were recovered and least $12(8.6 \%)$ cases were recovered within the duration of 5 -days. (table 5 )

Table 5: Association of time in recovery of acidosis

\begin{tabular}{|l|l|l|}
\hline Variables & Frequency & \%age \\
\hline Time & 15 & 21.43 \\
\hline Day & 24 & 34.3 \\
\hline 2-days & 15 & 21.43 \\
\hline 3-days & 14 & 20 \\
\hline 4-days & 12 & 17.14 \\
\hline$>$ 5days &
\end{tabular}

There was no any mortality found in this study.

\section{DISCUSSION}

This study was done to assess the need for intravenous liquid and insulin to overcome the crisis in the management of DKA.In this cross-sectional study total 70 patients were presented. Majority of the patients were females $54.3 \%$. In a previous study in Taipei,2 it was found that $67 \%$ DKA patients were female. Mean age of the patients was $30.51 \pm 7.41$ years with mean BMl $25.18 \pm 3.48 \mathrm{~kg} / \mathrm{m}^{2}$. This was comparable to the previous study.

In present study $42(60 \%)$ cases had previous history of diabetes and the rest $28(40 \%)$ were newly diagnosed. Prevalence of DKA was more common in low socioeconomic status found in $39(55.7 \%)$ cases. Hussain et in 2020 presented the same outcomes. [15] Vomiting was the most common symptom found in $35(50 \%)$ cases, followed by infection of insulin doses 18 (25.7\%) cases. The analysis in Atlanta and Pakistan produced comparable results. $[16,17]$ Most patients showed normal or slightly low 
levels of $\mathrm{Na}+$, high levels of normal or significantly higher $\mathrm{K}+$.

It took 48 hours for most patients. The acidosis correction took 2 days for the maximum. In prior investigations, this time was 12 to 15 hours. [18,19] In our study most cases $25.71 \%$ needed $91-100$ units of insulin. Each patient needs roughly 4.12 liters of IV fluid in developing countries, 72 insulin units and $60 \mathrm{mmol}$ of potassium during the first 24 hours of hospital therapy. [20] The results were slightly satisfactory and similar to those of industrialized countries. 5 (10 percent) individuals were expired with serious acidity.[21] Mortality was $6-24 \%$ in developing country but in our study there was 0 any mortality found. No previous research compared clinic results with and without priming insulin bolus in pediatric DKA patients. Therefore, it is not suggested to utilize priming bolus in pediatric DKA treatment. An initial bolus could help to correct the relative resistance to insulin DKA, based on the study in individuals suffering from hyperosmolar hyperglycemia nonchaeotic diabetes. [22] Therefore, patient factors, such lack of ketosis or the presence of severe hyperglycemia, could have led to conflicting outcomes. In our perspective, with administration of intravenous fluids and alleviation of hyperglycemia the majority of patients with DKA can quickly become insulin sensitive. Therefore, the greater rates of insulin infusion should be reserved for obese and insulin-resistant DKA patients in order to prevent hypoglycemia and fast shifts in glucose and water between extracellular and intracellular compartments.

The early studies on optimal insulin dosages and the route of treatment of DKA showed that regular insulin subcutaneous application is successful, albeit lower than intravenous insulin infusion.[23]

\section{CONCLUSION}

We concluded in this study that early diagnosed of diabetic ketoacidosis is necessary for treatment. Removal of adequate fluid was necessary to avoid mortality.

\section{REFERENCE}

1. Rouf R, Rahim M, Latif ZA. Requirement of Intravenous Fluid and Insulin in the Management ofDiabetic Ketoacidosis to Overcome the Crisis: Experience in a Specialized Hospital. Journal of Medicine.2018;19(1):18.

2. French EK, Donihi AC, Korytkowski MT. Diabetic ketoacidosis and hyperosmolar hyperglycemicsyndrome: review of acute decompensated diabetes in adult patients. Bmj. 2019 May 29;365.

3. Rouf $R$, Rahim MA, Latif ZA. The requirement of insulin and intravenous fluid in adult Diabeticketoacidosis management: a review. Open Access J Trans Med Res. 2018;2(5):132.

4. Brown HD, Tran RH, Patka JH. Effect of Bolus Insulin Administration Followed by a ContinuousInsulin Infusion on Diabetic Ketoacidosis Management. Pharmacy. 2018 Dec;6(4):129.

5. Varela D, Held N, Linas S. Overview of cerebral edema during correction of hyperglycemic crises.The American journal of case reports. 2018;19:562.

6. von Oettingen JE, Rhodes ET, Wolfsdorf Jl. Resolution of ketoacidosis in children with new onsetdiabetes: evaluation of various definitions. Diabetes research and clinical practice. 2018 Jan 1;135:76-84

7. Oray NC, Bayram B, Altintas E, Sivrikaya S, Savran Y Severe allergic reaction to human insulin inthe patient with diabetic ketoacidosis. Turkish journal of emergency medicine. 2018 Mar 1;18(1):40-1.

8. Broadley LK, Clark K, Ritchie GL. Prevention and management of hyperglycaemic crisis. NursingStandard. 2019 Sep 4;34(9):75-82.

9. Gupta D, Prasad A, Siddiqui F, Sitaula S, Albert SG. Insulin infusion responses in diabetic ketoacidosisalone and with a mixed hypochloremic alkalosis. Diabetes \&amp; Metabolic Syndrome: Clinical Research \&amp;Reviews. 2019 Jan 1;13(1):216-21.

10. Dingle HE, Slovis C. Diabetic ketoacidosis and hyperosmolar hyperglycemic syndrome management.Emerg Med. 2018;50(8):161-71.

11. Usman A. Initial potassium replacement in diabetic ketoacidosis: the unnoticed area of gap. Frontiersin endocrinology. 2018 Mar 21;9:109.

12. Dhatariya K, Savage M, Sampson M, Matfin G, Scott A. Severe hyperglycemia, diabetic ketoacidosis, and hyperglycemic hyperosmolar state. Endocrine and Metabolic Medical Emergencies: A Clinician\&\#39;sGuide. 2018 Mar 2:531- 47

13. Lin SF, Lin JD, Huang YY. Diabetic ketoacidosis: comparisons of patient characteristics, clinicalpresentations and outcomes today and 20 years ago. Chang Gunh Med J 2005;28(1):24-30

14. Jahagirdar RR, Khadilkar VV, Khadilkar AV, Lalwani SK. Management of diabetic ketoacidosis inPICU. Indian $\mathrm{J}$ Pediatr 2007;74(6):551-4.

15. Shabir Hussain, Amjad Mustafa , Arif Mumtaz, Ghazala Shaheen, Mohammad Soban, Shafiq Ahmad. Role of Insulin and Intravenous Fluid in the Treatment ofDiabetic Ketoacidosis to overt the Diabetic Ketoacidosis crisis. P J M H S Vol. 14, NO. 4, OCT - DEC2020

16. Murray-Bachmann R, O\&\#39;Flaherty D, Lewis-Holman S, Patterson SM, Ashman S, Macyk I. Safelmplementation of a Hyperglycemic Crises Protocol by Utilizing a Conversion Table

17. Abunga DK. An Audit Of The Management Of Diabetic Ketoacidosis At The Kenyatta NationalHospital (Doctoral dissertation, University of Nairobi)

18. Oliver WD, Willis GC, Hines MC, Hayes BD. Comparison of Plasma-Lyte A and Sodium Chloride $0.9 \%$ for Fluid Resuscitation of Patients With Diabetic Ketoacidosis. Hospital pharmacy. 20180ct;53(5):326-30

19. Rodriguez-Gutierrez $R$, Mancillas-Adame $L G$, RodríguezTamez G, Diaz Gonzalez-Colmenero A,SolisPacheco RC, Elizondo-Plazas AS, Santos-Santillana KM, GonzalezSariñana L, Gonzalez-Nava V,Gonzalez-Gonzalez JG. Hypertriglyceridemia and its association with $\mathrm{HbA1c}$ test: A prospective in vivocontrolled study. International journal of endocrinology. 2019 Feb 12;2019.

20. Wang CC, Zaman A. DIABETES MELLITUS: ACUTE AND CHRONIC COMPLICATIONS.Endocrine Secrets E-Book. 2019 Jul 16:12.

21. H.E. Lebovitz. Diabetic Ketoacidosis. The Lancet, 1995;345(8952):762-772

22. Rosenthal NR, Barrett EJ. An assessment of insulin action in hyperosmolar hyperglycemic nonketoticdiabetic patients. J Clin Endocrinol Metab. 1985;60(3):607-610

23. Fisher JN, Shahshahani MN, Kitabchi AE. Diabetic ketoacidosis: low-dose insulin therapy by variousroutes. N Engl J Med. 1977;297(5):238-241 